\section{DESIGUALDADES SOCIOECONÔMICAS E MORTALIDADE POR CÂNCER: UM ESTUDO ECOLÓGICO NO BRASIL}

\author{
Socioeconomic inequality and cancer mortality: an ecological \\ study in Brazil
}
Desigualdades socioeconómicas y mortalidad por cáncer: un estudio ecológico en Brasil

\section{RESUMO}

Objetivo: Analisar as desigualdades socioeconômicas e a mortalidade por câncer no Brasil. Métodos: Foram analisados os óbitos por câncer ocorridos entre 2010 e 2012, obtidos no Sistema de Informação sobre Mortalidade. Foram selecionados 268 municípios brasileiros que apresentaram população acima de 80 mil habitantes e melhor qualidade de informação. Os indicadores socioeconômicos referentes ao ano 2000 foram extraídos do Atlas Brasil 2013. Para analisar a correlação entre indicadores socioeconômicos e a mortalidade por câncer foi utilizada a Correlação de Pearson e a regressão linear simples. Resultados: Verificou-se correlação negativa com o analfabetismo $(\mathrm{r}=-0,509)$ e com o Gini $(\mathrm{r}=-0,197)$; a correlação foi positiva com o indicador de renda $(r=0,414)$ e esperança de vida $(r=0,537)$; a regressão linear simples mostrou que há uma associação fraca entre a mortalidade por câncer e as variáveis socioeconômicas pesquisadas. Conclusão: A análise da mortalidade por câncer nos municípios brasileiros mostrou que as maiores taxas de mortalidade foram registradas nos municípios com as melhores condições socioeconômicas, expressadas pelos indicadores de renda e esperança de vida.

Descritores: Neoplasias; Mortalidade; Fatores Socioeconômicos; Desigualdades em Saúde.

\section{ABSTRACT}

Objective: To analyze the socioeconomic inequalities and cancer mortality in Brazil. Methods: The study analyzed the deaths for cancer occurred from 2010 to 2012, obtained in the Mortality Information System. A total of 268 Brazilian municipalities presenting population over 80,000 inhabitants and better quality of information were selected. Socioeconomic indicators for the year 2000 were taken from the Atlas Brazil 2013. Pearson correlation and simple linear regression were applied to investigate the correlation between socioeconomic indicators and cancer mortality. Results: There was a negative correlation with illiteracy ( $r=-0.509)$ and the Gini coefficient ( $r=-0.197)$; the correlation was positive with the income indicator $(r=0.414)$ and life expectancy $(r=0.537)$; simple linear regression showed that there is a weak association between cancer mortality and the socioeconomic variables assessed. Conclusion: The cancer mortality analysis in Brazilian municipalities showed that the highest mortality rates were recorded in the municipalities with the best socioeconomic conditions, expressed by indicators of income and life expectancy.

Descriptors: Neoplasms; Mortality; Socioeconomic Factors; Health Inequalities.
Artigo Original
1) Universidade Federal do Rio Grande do Norte - UFRN - Natal - Brasil

2) Universidad de Zaragoza - UNIZAR Zaragoza - Espanha
Recebido em: 15/02/2016 Revisado em: 06/04/2016 Aceito em: 13/05/2016 


\section{RESUMEN}

Objetivo: Analizar las desigualdades socioeconómicas y la mortalidad por cáncer en Brasil. Métodos: Fueron analizados los óbitos por cáncer entre 2010 y 2012 identificados en el Sistema de Información sobre Mortalidad. Fueron elegidos 268 municipios brasileños que presentaron población mayor que 80 mil habitantes y mejor calidad de información. Los indicadores socioeconómicos referentes al año 2000 fueron retirados del Atlas Brasil 2013. Se utilizó la Correlación de Pearson y la regresión linear simple para analizar la correlación entre los indicadores socioeconómicos y la mortalidad por cáncer. Resultados: Se verificó una correlación negativa con el analfabetismo $(r=-0,509)$ y con el Gini $(r=-$ 0,197); la correlación fue positiva para el indicador de renta $(r=0,414)$ y la esperanza de vida $(r=0,537)$; la regresión linear simple mostró una asociación débil entre la mortalidad por cáncer y las variables socioeconómicas investigadas. Conclusión: El análisis de la mortalidad por cáncer en los municipios brasileños mostró que las mayores tasas de mortalidad se registraron en los municipios con mejores condiciones socioeconómicas expresadas por los indicadores de renta y esperanza de vida.

Descriptores: Neoplasias; Mortalidad; Factores Socioeconómicos; Desigualdades en Salud.

\section{INTRODUÇÃO}

O câncer é a principal causa de morte em países desenvolvidos e, em poucas décadas, se tornará a principal causa de morbidade e mortalidade nas regiões mais pobres

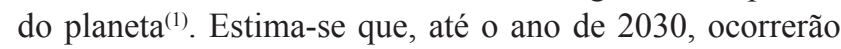
22,2 milhões de casos de câncer e 12,7 milhões de mortes em todo o mundo. Dois terços dessas mortes ocorrerão nos países pobres e em desenvolvimento ${ }^{(2)}$.

O envelhecimento da população, a adoção de estilos de vida associados ao desenvolvimento do câncer, a desestruturação dos serviços de saúde bem como os fatores relacionados às iniquidades sociais fazem com que haja uma incoerência de que tanto a incidência como a mortalidade por neoplasias malignas sigam aumentando nos países em desenvolvimento apesar dos avanços no diagnóstico e tratamento do câncer em anos recentes ${ }^{(3)}$.

As desigualdades na carga de câncer podem ser observadas entre os países bem como dentro de um mesmo país ${ }^{(4)}$. Os modelos conceituais relacionados a fatores socioeconômicos e de estruturação dos serviços de saúde, incluindo aspectos relacionados aos diferentes níveis de exposição a fatores de risco e de acesso a diagnóstico, tratamento e prevenção, explicam as iniquidades na mortalidade por câncer entre os grupos socioeconômicos, étnicos e de gênero ${ }^{(5)}$.
A estimativa para o Brasil, para o biênio 2016-2017, aponta a ocorrência de cerca de 600 mil casos novos de câncer. Excetuando-se o câncer de pele não melanoma (aproximadamente 180 mil casos novos), ocorrerão cerca de 420 mil casos novos de câncer nos quais figurarão como os tipos mais frequentes em homens o câncer de próstata $(28,6 \%)$, pulmão $(8,1 \%)$ e intestino $(7,8 \%)$, e nas mulheres, os cânceres de mama $(28,1 \%)$, intestino $(8,6 \%)$ e colo do útero $(7,9 \%)^{(6)}$.

As transições demográfica e epidemiológica têm contribuído para a mudança no perfil do risco para as doenças crônicas como o câncer. Os níveis de consumo de tabaco, os padrões da dieta, e as características reprodutivas, além da prevalência das infecções relacionadas ao câncer, são padrões que têm mudado rapidamente. Como resultado desse processo, o câncer destaca-se como a segunda causa de morte depois das doenças cardíacas e cerebrovasculares no Brasil ${ }^{(7)}$.

O resultado das elevadas incidências, principalmente dos cânceres relacionados às infecções, associadas à desestruturação dos sistemas de cuidado ao paciente com câncer, em que as intervenções para detecção precoce e tratamentos efetivos permanecem inacessíveis para a maioria das pessoas, resultam em elevadas taxas de mortalidade nos países pobres ou em desenvolvimento, a exemplo do Brasil ${ }^{(8)}$.

O Brasil está no ranking entre os países com as maiores desigualdades sociais e econômicas do mundo, e apesar do substancial desenvolvimento econômico e social vivido pelo país nas últimas décadas, as profundas desigualdades sociais aliadas à falta de um planejamento abrangente do sistema de saúde têm levado a exacerbação das iniquidades no acesso aos cuidados em saúde ${ }^{(9)}$. A conjunção desses problemas é crucial na determinação dos níveis de mortalidade por câncer, já que os casos fatais são influenciados pelo diagnóstico precoce e a disponibilidade de tratamentos e cuidados ao paciente ${ }^{(5)}$.

As evidências demonstram que os grupos de níveis socioeconômicos mais baixos têm apresentado elevada mortalidade por câncer em geral devido a maior proporção de diagnóstico tardio de neoplasias passíveis de detecção em estágios iniciais por meio de rastreamento; maior dificuldade de acesso ao diagnóstico e tratamento adequado; pior prognóstico e menor sobrevida após o diagnóstico de câncer; maior risco de óbito por câncer em geral e por tipos de câncer potencialmente curáveis ${ }^{(8)}$. Destaca-se, dessa forma que, na linha de cuidado do câncer, a atenção primária à saúde tem importante destaque quanto a ações de promoção, prevenção, detecção precoce e cuidados paliativos. 
A produção de estudos ecológicos que analisem os padrões sociais e econômicos permite-nos quantificar as disparidades na mortalidade por câncer relacionado aos grupos em vantagem ou desvantagem social e identificar áreas ou grupos populacionais que estão sob maior risco de mortalidade por câncer, servindo de informação para o monitoramento do estado de saúde das populações, para a identificação de grupos de risco, para o planejamento, definição e implantação de políticas públicas voltadas para as áreas mais vulneráveis.

Baseado na importância que a carga de câncer apresenta na saúde da população brasileira, frente às intensas desigualdades sociais registradas no país, o objetivo desse estudo foi analisar as desigualdades socioeconômicas e a mortalidade por câncer no Brasil.

\section{MÉTODOS}

Trata-se de um estudo ecológico de múltiplos grupos cujas unidades de análise foram os municípios brasileiros selecionados a partir de critérios demográficos e de qualidade dos registros de mortalidade.

$\mathrm{O}$ critério para seleção dos municípios considerou o problema da variabilidade das taxas de mortalidade (que diz respeito à flutuação anual que as taxas de mortalidade sofrem devido às pequenas populações) e da qualidade da informação (devido ao sub-registro de óbitos e às deficiências na certificação das causas de morte no Brasil).

Para minimizar o problema da variabilidade das taxas de mortalidade, foram inicialmente selecionados 357 municípios que possuíam população igual ou superior a 80.000 habitantes no ano de 2010.

Procurou-se assegurar melhor qualidade do registro das causas de óbito admitindo-se uma proporção máxima de $10 \%$ de causas mal definidas, na média para os anos de 2010 a 2012. Esse processo resultou na seleção de 268 municípios, representados por 118 municípios da região sudeste, 56 municípios da região nordeste, 52 municípios da região sul, 25 do centro oeste e 17 da região norte.

A variável dependente foi a mortalidade por câncer, expressada pela Taxa de Mortalidade Padronizada (TMP). Foram consideradas como variáveis independentes os indicadores socioeconômicos representados pelo Coeficiente de Gini, a Renda per capita, a Esperança de vida ao nascer e a Taxa de analfabetismo de pessoas acima de 25 anos.

Para o cálculo das taxas de mortalidade por câncer foram utilizados os óbitos decorrentes do conjunto das neoplasias malignas ocorridas no Brasil no período de 2010 a 2012. Os óbitos foram obtidos de forma secundária coletados do Sistema de Informação sobre Mortalidade
(SIM) constante no sítio eletrônico do Ministério de Saúde do Brasil.

Os dados de população por município foram obtidos das informações do Censo 2010 e das projeções intercensitárias, no sítio do Instituto Brasileiro de Geografia e Estatística ${ }^{(10)}$. Os indicadores socioeconômicos para o ano 2000 foram coletados do Atlas de Desenvolvimento Humano no Brasil 2013, também denominado Atlas Brasil 2013, do Programa das Nações Unidas para o Desenvolvimento (PNUD) ${ }^{(11)}$.

As taxas brutas foram padronizadas pelo método direto, considerando a população padrão mundial e expressas por 100.000 habitantes por ano ${ }^{(12)}$.

Foi procedida à análise descritiva das variáveis utilizadas no estudo; e para avaliar a correlação entre os indicadores socioeconômicos selecionados e a mortalidade por câncer no Brasil foram aplicados os testes de correlação de Pearson e a regressão linear simples. Para o processamento e a análise estatística, foi utilizado o programa IBM SPSS Statistics 22.0.

Essa pesquisa utilizou dados secundários disponíveis em sites oficiais do Ministério da Saúde do Brasil sendo dispensado de apreciação em comitê de ética em pesquisa, em conformidade com a Resolução 466112 do Conselho Nacional de Saúde.

\section{RESULTADOS}

A tabela I apresenta a descrição das variáveis socioeconômicas pesquisadas e a média das taxas de mortalidade por câncer nos 268 municípios selecionados, agrupados por regiões brasileiras. Observa-se que as piores condições sociais e econômicas estão concentradas nas regiões norte e nordeste do Brasil, sendo esta última a que apresentou os piores indicadores de renda per capta $(\mathrm{R} \$ 364,84)$ e maior percentual de pessoas analfabetas $(24,12 \%)$. Nas regiões norte e nordeste, os municípios selecionados possuem, em média, esperança de vida de menos de 70 anos, mais de $15 \%$ da população acima de 25 anos analfabeta, e com índice de Gini em torno de 0.6.

As regiões mais desenvolvidas do país, sul e sudeste, apresentam grandes diferenças em relação aos mais pobres. Essas regiões apresentaram uma renda per capta em torno de $\mathrm{R} \$ 720$ e taxa de analfabetismo menor que $10 \%$.

Por outro lado, as maiores taxas de mortalidade por câncer foram registradas nos municípios da região sul e sudeste do país, que registraram uma média de 94,65 óbitos $/ 100$ mil habitantes no Sudeste e 116 óbitos $/ 100$ mil habitantes na região sul do Brasil.

A correlação entre as variáveis independentes e dependentes mostrou uma correlação forte e inversamente proporcional com o analfabetismo $(\mathrm{r}=-0,595)$. A correlação 
Tabela I - Valores médios das variáveis socioeconômicas e da mortalidade por câncer de 268 municípios brasileiros por região do país. Brasil, 2015.

\begin{tabular}{lccccc}
\hline Variáveis & Norte & Nordeste & Centro Oeste & Sudeste & Sul \\
\hline Taxa de mortalidade por câncer padronizada $^{1}$ & 82,64 & 81,45 & 89,85 & 94,65 & 116,04 \\
Renda per capta $^{2}$ & 428,31 & 364,84 & 580,92 & 730,54 & 725,82 \\
Esperança de vida $^{3}$ & 68,92 & 67,88 & 71,47 & 72,10 & 73,70 \\
Analfabetismo $^{4}$ & 15,68 & 24,12 & 13,26 & 9,40 & 7,68 \\
Gini & 0,60 & 0,58 & 0,56 & 0,54 & 0,53 \\
\hline
\end{tabular}

${ }^{1}$ Taxa padronizada de mortalidade por câncer expresso em número de óbitos por 100 mil habitantes; ${ }^{2}$ Renda per capita expressa em Reais (R\$); ${ }^{3}$ Esperança de vida expressa em anos; ${ }^{4}$ Analfabetismo de pessoas acima de 25 anos expresso em percentual de pessoas analfabetas em relação ao total da população.

Tabela II - Relação entre as variáveis socioeconômicas e a mortalidade padronizada por câncer nos 268 municípios brasileiros selecionados. Brasil, 2015.

\begin{tabular}{lccccc}
\hline Variáveis & TMP & Renda & Esperança de Vida & Analfabetismo & Gini \\
\hline TMP & 1 & & & & \\
Renda & $0,414^{* *}$ & 1 & & & \\
Esperança de vida & $0,537^{* *}$ & $0,622^{* *}$ & 1 & 1 & \\
Analfabetismo & $-0,595^{* *}$ & $-0,687^{* *}$ & $-0,745^{* *}$ & $0,202^{* *}$ & 1 \\
Gini & $-0,197^{* *}$ & $0,163^{* *}$ & $-0,254^{* *}$ & \\
\hline
\end{tabular}

Fonte: Censo Demográfico, 2000/IBGE; SIM/Datasus/Ministério da saúde; ${ }^{*} \mathrm{p}<0,05,{ }^{*} \mathrm{p}<0,01$; TMP: Taxa de mortalidade padronizada.
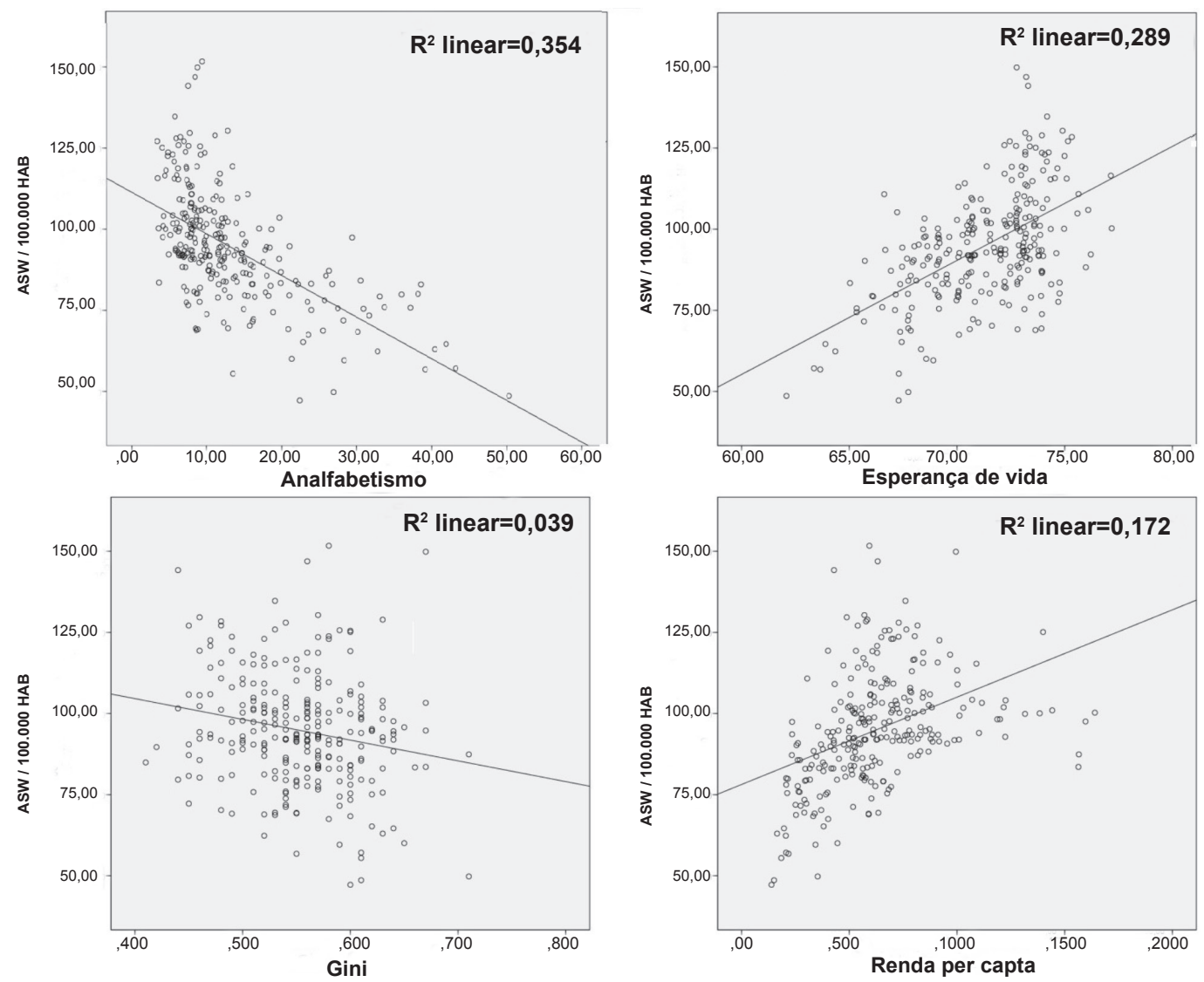

Figura 1 - Regressão linear simples entre a taxa padronizada de mortalidade por câncer e os indicadores socioeconômicos dos 268 municípios brasileiros selecionados. Brasil, 2015. 
foi positiva com os indicadores de renda $(\mathrm{r}=0,414)$ e esperança de vida $(r=0,537)$ (Tabela II).

A análise de regressão simples mostrou que a melhor condição socioeconômica está associada a maiores riscos de mortalidade por câncer. $\mathrm{O}$ indicador de analfabetismo foi o único que apresentou associação com a variável dependente, embora essa associação seja considerada fraca $\left(\mathrm{r}^{2}=0,354\right)($ Figura 1$)$

\section{DISCUSSÃO}

Assim como no presente trabalho, outros estudos também evidenciaram a correlação positiva entre a mortalidade por câncer e as melhores condições socioeconômicas $^{(13-15)}$. O estudo que avaliou a mortalidade por câncer de cólon e reto no Brasil observou uma correlação direta com os indicadores de renda bruta ${ }^{(13)}$. No Irã, foi observada correlação positiva entre a mortalidade por câncer de mama e ovários e o indicador de ranking social das províncias ${ }^{(14)}$. Elevadas taxas de mortalidade por câncer foram registradas nas populações com mais elevado nível de educação em países como a Espanha, Finlândia, Dinamarca, Noruega, Bélgica, França, Suíça e Áustria ${ }^{(15)}$.

Mais recentemente, foi descrita a transição global do câncer de acordo com a estratificação do Índice de Desenvolvimento Humano (IDH). Essa transição sugere que haverá redução dos casos de câncer relacionados às infecções (por exemplo, à infecção pelo HPV- Human Papiloma Virus) enquanto haverá aumento dos casos de câncer associado aos fatores de risco comuns às doenças crônicas não-transmissíveis. Os cânceres de mama, pulmão, color retal e próstata estão relacionados aos países com mais elevados IDH enquanto o câncer cervical está relacionado aos países menos desenvolvidos ${ }^{(1)}$. Essa hipótese poderia explicar os resultados de pesquisas brasileiras que indicaram correlação inversa entre as condições socioeconômicas e a mortalidade por alguns tipos de câncer que estão relacionados às baixas condições socioeconômicas, como o câncer de boca ${ }^{(16)}$, cabeça e pescoço ${ }^{(17)}$.

Essa ideia é reforçada pelos achados apresentados na revisão de estudos ecológicos que relacionam a incidência e a mortalidade por câncer e as desigualdades socioeconômicas: a incidência de câncer de próstata e mama e a mortalidade por câncer de cólon são positivamente correlacionadas com o nível socioeconômico da área de moradia. Por outro lado, uma correlação consistente e negativa foi encontrada para a incidência e mortalidade por cânceres de esôfago, estômago e colo uterino ${ }^{(18)}$.

É possível que o problema do sub-registro diferencial de óbitos e a qualidade da informação sobre causas de morte interfiram nos resultados das análises de mortalidade em estudos ecológicos no Brasil. No presente trabalho, a utilização de critérios que assegurassem a seleção de municípios com melhor qualidade da informação resultou em achados compatíveis com os de estudos internacionais nos quais a qualidade de informação está garantida ${ }^{(15,19)}$.

A constatação de que há correlação direta entre a mortalidade por câncer e as melhores condições de vida no Brasil trazem à tona o dilema do paradoxo brasileiro, sublinhado nos padrões epidemiológicos da mortalidade por câncer e nas condições sociais e econômicas das distintas populações no país. Considerado como uma das maiores economias emergentes do planeta e apesar dos avanços econômicos, sociais e políticos pelos quais passou o país na última década, as desigualdades na distribuição da renda ao longo da história produziu abismos sociais marcantes ${ }^{(20)}$, reproduzidos, por exemplo, nos indicadores sociais como o IDH dos municípios brasileiros. A exacerbação das desigualdades internas é tão relevante que se pode encontrar no Brasil áreas com IDH de países desenvolvidos, como a Dinamarca e a Irlanda (IDH $>0,8$ ), assim como áreas com IDH de países africanos, como a Etiópia e o Congo $(\mathrm{IDH}<0,4)^{(21)}$.

O paradoxo brasileiro é expresso também nos padrões epidemiológicos da mortalidade por câncer. Além da superposição entre as etapas da transição epidemiológica, que combina elevadas taxas de morbidade e mortalidade por doenças crônico-degenerativas com altas incidências de doenças infecciosas e parasitárias, a polarização epidemiológica é reproduzida por meio da persistência de níveis diferenciados de transição entre grupos sociais distintos ${ }^{(22)}$, representada através das diferenças nas taxas de mortalidade por câncer entre as regiões brasileiras, como foi observado neste estudo.

No Brasil, os cânceres mais incidentes e que mais provocam óbitos na população são aqueles relacionados com elevados níveis de condições de vida, como os cânceres de pulmão, próstata e mama, em contraponto ao câncer cervical e de estômago, característicos de áreas com condições socioeconômicas menos privilegiadas ${ }^{(23)}$. A partir desse perfil, o paradoxo brasileiro pode ser descrito pelo quadro de um país subdesenvolvido com características de mortalidade por câncer de um país desenvolvido, o que pode explicar a correlação entre melhores condições de vida e maior mortalidade por câncer.

Os indicadores selecionados para este estudo refletem de forma geral a situação socioeconômica de uma população podendo ser considerados como causa e consequência da pobreza, determinando os modos de nascer, viver e de morrer de uma população ${ }^{(9)}$. O modelo utilizado para compreensão dos Determinantes Sociais da Saúde neste estudo é o modelo que inclui as condições naturais e sociais que afetam a saúde e os mecanismos que através dos quais essas condições produzem esses efeitos, incluindo além das 
condições sociais e econômicas mais gerais, o desemprego, as condições de moradia, o nível educacional e o acesso aos serviços de saúde como variáveis mais proximais ${ }^{(24)}$.

Três causas principais podem estar relacionadas com as diferenças nas taxas de mortalidade por câncer em uma população definida: a prevalência e distribuição dos fatores de risco relacionados ao câncer ${ }^{(17)}$, a conformação da rede de serviços de saúde ${ }^{(20)}$, e a estrutura demográfica do país ${ }^{(22)}$. Os determinantes sociais e econômicos influenciam esses três pontos chave que estão relacionados com os padrões de mortalidade por câncer.

O tabagismo, o alcoolismo, as características reprodutivas e sexuais, a prevalência das infecções relacionadas ao câncer, o uso de terapias hormonais, os padrões alimentares, a atividade física e os comportamentos relacionados ao estilo de vida ocidentalizado e de alta renda, são descritos como fatores de risco ao desenvolvimento do câncer, e que por sua vez, esses comportamentos são intimamente determinados pelo nível de condição social e econômica dos indivíduos ${ }^{(7,8,16,18)}$.

A estruturação dos serviços de screening, da realização das ações profiláticas de imunização e do controle dos fatores de risco, a disponibilidade e o acesso aos métodos diagnósticos e de tratamento para o câncer afetam as taxas de sobrevivência e de mortalidade por câncer numa população ${ }^{(19)}$. No Brasil, assim como em outros países latino-americanos, existe uma marcante iniquidade entre regiões, e entre os grandes centros urbanos e o interior, quanto ao acesso a serviços de diagnóstico e tratamento de câncer ${ }^{(25)}$. No presente estudo, a associação da mortalidade por câncer em populações mais privilegiadas e que tem maior acesso aos recursos diagnósticos e terapêuticos pode ser resultado, inclusive, da melhor estruturação dos serviços de vigilância epidemiológica do câncer nessas áreas, o que resulta na melhor qualidade nos registros de óbito.

Nesse sentido, parece urgente a implantação de políticas públicas de saúde direcionadas às populações mais afetadas, associadas à diminuição das iniquidades sociais e de acesso à prevenção primária, ao diagnóstico precoce e a tratamentos no intuito de reduzir as disparidades na mortalidade por câncer no Brasil.

Destaca-se como uma limitação deste estudo a utilização de dados secundários sobre mortalidade que está sujeito à subnotificação, apesar de nos últimos anos reconhecer-se que o Sistema sobre Mortalidade no Brasil obteve um ganho significativo de qualidade.

\section{CONCLUSÃO}

Os resultados encontrados indicam que existe uma desigualdade na distribuição da mortalidade por câncer no
Brasil influenciada por fatores sociais e econômicos. As regiões sul e sudeste do país registraram as maiores taxas de mortalidade e os melhores indicadores socioeconômicos, mostrando que no Brasil, a mortalidade apresenta correlação direta com as melhores condições de vida, expressas pelos indicadores de renda e esperança de vida.

\section{FINANCIAMENTO}

Programa de Doutorado Sanduíche no Exterior (PDSE) (processo $\mathrm{n}^{\circ}$. 99999.002059/2014-02) da Coordenação de Aperfeiçoamento de Pessoal de Nível Superior (CAPES) e Edital Universal do Conselho Nacional de Desenvolvimento Científico e Tecnológico (CNPq) (processo $n^{\circ}$. 481805/ 2013-3).

\section{REFERÊNCIAS}

1. Bray F, Jemal A, Grey N, Ferlay J, Forman D. Global cancer transitions according to the Human Development Index (2008-2030): a population-based study. Lancet Oncol. 2012;13(8):790-801.

2. Farmer P, Frenk J, Knaul FM, Shulman LN. Expansion of cancer care and control in countries of low and middle income: a call to action. Lancet. 2010; 376(9747):1186-93.

3. Parkin DM. The role of cancer registries in cancer control. Int J Clin Oncol. 2008;13(2):102-11.

4. Barbosa IR, Souza DLB, Costa ICC, Pérez MB. Cancer mortality in Brazil. Medicine (Baltimore). 2015;94(16):e746.

5. Puigpinós R, Borrell C, Antunes JLF, Azlor E, Pasarín MI, Serral G, et al. Trends in socioeconomic inequalities in cancer mortality in Barcelona: 1992-2003. BMC Public Health. 2009;9:35.

6. Instituto Nacional de Câncer José Alencar Gomes da Silva. Estimativa 2016: incidência de câncer no Brasil. Rio de Janeiro: INCA; 2015.

7. Barbosa IR, Costa ICC, Bernal MM, Souza DLB. Tendência das taxas de mortalidade pelas dez principais causas de óbitos por câncer no Brasil, 1996-2012. Rev Ciência Plural. 2016;2(1):3-16.

8. Barbosa IR, Costa ICC, Bernal MM, Souza DLB. As iniquidades sociais e as disparidades na mortalidade por câncer relativo ao gênero. Rev Ciência Plural. 2015;1(2):79-86.

9. Pisani P. The cancer burden and cancer control in developing countries. Environ Health. 2011;10(Supp11):S2. 
10. Instituto Brasileiro de Geografia e Estatística - IBGE. Estimativas da população [acesso em 2014 Jul 20]. Disponível em: http://www.ibge.gov.br/home/ estatistica/populacao/estimativa2015/default.shtm

11. Programa das Nações Unidas para o Desenvolvimento. Atlas do Desenvolvimento Humano do Brasil: consulta [acesso em 2014 Jul 20]. Disponível em: http://www. atlasbrasil.org.br/2013/pt/consulta/

12. Doll R, Payne P, Waterhouse JA, editors. Cancer Incidence in Five Continents. Berlin: Springer; 1966.

13. Guimarães RM, Rocha PGM, Muzi CD, Ramos RS. Incremento de renda e mortalidade por câncer colorretal no Brasil, 2001-2009. Arq Gastroenterol. 2013;50(1):64-9.

14. Kiadaliri AA. Social disparity in breast and ovarian cancer incidence in Iran, 2003-2009: a time trend province-level study. J Breast Cancer. 2013;16(4): 372-77.

15. Strand BH, Kunst A, Huisman M, Menvielle G, Glickman M, Bopp M, et al. The reversed social gradient: higher breast cancer mortality in the higher educated compared to lower educated. A comparison of 11 European populations during the 1990s. Eur J Cancer. 2007;43(7):1200-7.

16. Ferreira MAF, Gomes MN, Michels FAS, Dantas AA, Latorre MRDO. Desigualdade social no adoecimento e morte por câncer de boca e orofaríngeo no Município de São Paulo, Brasil: 1997 a 2008. Cad Saúde Pública. 2012;28(9):1663-73.

17. Antunes JLF, Toporcov TN, Biazevic MGH, Boing AF, Bastos JL. Gender and racial inequalities in trends of oral cancer mortality in Sao Paulo, Brazil. Rev Saúde Pública. 2013;47(3):470-8.

18. Ribeiro AA, Nardocci AC. Desigualdades socioeconômicas na incidência e mortalidade por câncer: revisão de estudos ecológicos, 1998-2008. Saúde Soc. 2013;22(3):878-91.
19. Sharpe KH, McMahon AD, Raab GM, Brewster DH, Conway DI. Association between Socioeconomic Factors and Cancer Risk: A Population Cohort Study in Scotland (1991-2006). PLoS ONE. 2014;9(2):e89513.

20. Barros MBA, Franscisco PMSB, Zanchetta LM, César CLG. Tendências das desigualdades sociais e demográficas na prevalência de doenças crônicas no Brasil, PNAD: 2003- 2008. Ciênc Saúde Coletiva. 2011;16(9):3755-68.

21. United Nations Developments Program - UNDP. Human development reports [acesso em 2014 Jun 30]. Disponível em: www.hdr.org/in/data

22. Schramm JMA, Oliveira AF, Leite IC, Valente JG, Gadelha AMJ, Portela MC, et al. Transição epidemiológica e o estudo de carga de doença no Brasil. Ciênc Saúde Coletiva. 2004;9(4):897-908.

23. Ferlay J, Steliarova-Foucher E, Lortet-Tieulent J, Rosso S, Coebergh JW, et al. Cancer incidence and mortality patterns in Europe: estimates for 40 countries in 2012. Eur J Cancer. 2013;49(6):1374-403.

24. Graham H. Health Inequalities, social determinants and public health policy. Policy Polit. 2009;37(4):463-79.

25. Goss PE, Lee BL, Badovinac-Crnjevic T, StrasserWeippl K, Chavarri-Guerra Y, St Louis J, et al. Planning cancer control in latin america and the caribbean. Lancet Oncol. 2013;14(5):391-436.

\section{Endereço para correspondência:}

Isabelle Ribeiro Barbosa

Universidade Federal do Rio Grande do Norte - UFRN

Rua Princesa Leopoldina, 3466

Bairro: Candelária

CEP: 59065-100 - Natal - RN - Brasil

E-mail: isabelleribeiro@oi.com.br 